\title{
GCF Adrenomedullin Levels in Healthy and Periodontitis Patients with or without Type 2-Diabetes Mellitus: Clinicobiochemical Study
}

\author{
${ }^{1}$ Akanksha Garg, ${ }^{2} \mathrm{~A}$ Suchetha, ${ }^{3} \mathrm{~N}$ Sapna, ${ }^{4}$ SM Apoorva, ${ }^{5}$ Divya Bhat, ${ }^{6}$ Lakshmi Puzhankara
}

\begin{abstract}
Background: The literature suggests that periodontal disease and diabetes mellitus share a two-way relationship. The aim of this study was to evaluate and compare the levels of adrenomedullin (ADM) in gingival crevicular fluid (GCF) of periodontally healthy and periodontitis patients with or without type 2 diabetes with different glycemic controls.
\end{abstract}

Methods: Ninety patients were included in the study and were divided into five groups based on CPI scores and ADA classification of diabetes. Probing pocket depth (PPD) and clinical attachment level (CAL) were measured in all the subjects. GCF was collected from all the participants using micropipettes. ADM levels were measured in GCF samples by enzyme-linked immunosorbent assay.

Results: The results showed higher levels of ADM in patients with periodontitis as compared to healthy group. Significant correlation was present between PPD and CAL and ADM levels in all periodontitis patients with or without type 2 diabetes.

Conclusion: Increase in GCF levels of ADM from periodontal health to disease and in periodontitis patients with type 2 diabetes with the worsening of glycemic control underlines the possible role of ADM in mounting a protective response to worsening disease state.

Keywords: Diabetes mellitus, Adrenomedullin, Gingival crevicular fluid, Periodontitis, Glycated hemoglobin.

How to cite this article: Garg A, Suchetha A, Sapna N, Apoorva SM, Bhat D, Puzhankara L. GCF Adrenomedullin Levels in Healthy and Periodontitis Patients with or without Type 2-Diabetes Mellitus: Clinicobiochemical Study. World J Dent 2014;5(1):42-46.

Source of support: Nil

Conflict of interest: None

\section{INTRODUCTION}

Periodontal diseases are a group of lesions affecting the tissues surrounding and supporting the teeth in their sockets. ${ }^{1}$ The interactions between the host defence

\footnotetext{
${ }^{1,6}$ Postgraduate Student, ${ }^{2}$ Professor and Head, ${ }^{3,4}$ Reader ${ }^{5}$ Senior Lecturer

${ }^{1-6}$ Department of Periodontics, DAPM RV Dental College Bengaluru, Karnataka, India
}

Corresponding Author: Lakshmi Puzhankara, Postgraduate Student, Department of Periodontics, DAPM RV Dental College, Bengaluru, Karnataka, India, Phone: 08022445754 e-mail: lakshmi.p.menon83@gmail.com mechanism and microbial challenge are important factors for the susceptibility of the host to periodontitis, although the presence of microbial dental plaque is the primary etiologic factor for periodontal disease. ${ }^{2}$ Antimicrobial peptides including defensins, cathelicidin, and human neutrophil peptides (HNPs) participate in the innate host response of organisms, and they are important contributors for maintaining the balance between health and disease..$^{2,3}$ The role of antimicrobial peptides is especially important in the defence of the oral cavity because the oral cavity is constantly exposed to a variety of microbial pathogens. ${ }^{2}$

The oral epithelium plays a role as a physical barrier against pathogenic microorganisms, and it also contributes to the innate immune system. ${ }^{4}$ Adrenomedullin (ADM), which is constitutively expressed and secreted by oral epithelial cells, is one of the important antimicrobial peptides having a role in the innate host defence. ${ }^{5}$ Whole saliva contains higher concentrations of adrenomedullin than glandular saliva, suggesting that oral epithelial cells contribute to the salivary expression. ${ }^{6}$ In gingival crevicular fluid, the amount of ADM is about twice as high in periodontal disease sites than in healthy sites. ${ }^{5}$ Epithelial cells also secrete ADM when they are exposed to various bacterial species and inflammatory cytokines. ${ }^{5,7}$ In addition to the antibacterial role of ADM, it creates a feedback mechanism to activate the inflammatory response to pathogens. ${ }^{8}$ Adrenomedullin has vasodilator effects and might increase the numbers of inflammatory cells and mediators in inflamed gingival tissue. ${ }^{8}$

Periodontal disease and diabetes is like a two-way street. Research suggests that, as an infectious process with a prominent inflammatory component, periodontal disease can adversely affect the metabolic control of diabetes. Conversely, treatment of periodontal disease and reduction of oral inflammation may have a positive effect on the diabetic condition, although evidence for this remains somewhat equivocal. ${ }^{9}$

Studies have shown that plasma levels of ADM are increased in patients with type 2 diabetes mellitus as compared to controls and its levels have been found to be increased significantly in subjects with complications of type 2 diabetes mellitus. ${ }^{10}$ Studies have also reported 
that gingival crevicular fluid ADM concentrations are significantly higher in patients with periodontal disease.

This study was designed to evaluate and compare the adrenomedullin levels in periodontally healthy and periodontitis patients and its association with type 2 diabetes.

\section{METHODS}

\section{Source of Data}

The study population consisted of a total of 90 subjects in the age group of 35 to 75 years attending the outpatient section, Department of Periodontics, DA Pandu Memorial RV Dental College, Bengaluru, India, from December 2011 to May 2012. Written informed consent was obtained from all patients, and ethical clearance for the study was received from the Institutional Ethical Committee and Review Board, DAPMRV Dental College, Bengaluru, India. Patients of both sexes aged between 35 and 75 years with at least 3 teeth per quadrant were included in the study. Patients with systemic diseases, such as type 1 diabetes mellitus, cardiovascular disorder, immunologic disorders, hepatitis and human immunodeficiency virus infections were excluded, as were smokers, pregnant and lactating women and those taking oral contraceptive drugs or any anti-inflammatory or corticosteroids drugs. None of the subjects had received antibiotics within the previous 3 months or treatment for periodontal disease in the 6 months preceding the study.

The subjects were divided into five groups on the basis of their glycemic control and periodontal status.

\section{Criteria for Subject Grouping}

Group I (Healthy): 15 subjects with clinically healthy periodontium with no evidence of diabetes. CPI score ${ }^{11} 0$.

Group II: 30 subjects with a CPI score of 3 or more.

Group III: 15 diabetic subjects with CPI score of 3 or more. The HbA1c value: $>6$ and $<7$.

Group IV: 15 diabetic subjects with CPI score of 3 or more. The HbAlc value: $>7$ and $<8$.

Group V: 15 diabetic subjects with CPI score of 3 or more. The HbAlc value: $>8$.

All the participants underwent a detailed periodontal examination for the measurement of probing pocket depth (PPD) and clinical attachment level (CAL) using a University of North Carolina Probe (UNC-15 probe).

Test site for GCF sample collection was selected based on the site with highest CPI score in the oral cavity (periodontitis cases). In the healthy group, to standardize site selection and obtain adequate fluid volume, sampling was predetermined to be from the mesiobuccal region of the maxillary right first molar, in the absence of which the left first molar was sampled.

\section{Procedure for Sample Collection}

\section{Method of Collection of Blood}

The procedure was explained to the patient before collection of blood. The left antecubital fossa was swabbed with an alcohol swab and a cuff was used to apply pressure above the fossa. The blood was drawn with a $5 \mathrm{ml}$ syringe and immediately transferred to a vacutainer. $\mathrm{HbA} 1 \mathrm{c}$ was estimated by the turbidimetric inhibition assay (TINIA) method.

\section{Method of Collection of GCF}

Supragingival calculus, if present, was removed using Universal Scaler (Hu Friedy, Chicago, IL, USA). The test site was dried and isolated. Samples of GCF were obtained from predetermined sites by placing calibrated, volumetric, microcapillary pipettes (Sigma Aldrich Co., St. Louis, Missouri, USA) with 0-5 $\mu$ l range. The micropipettes were placed at the entrance of the gingival crevice. 2 to $3 \mu \mathrm{l}$ of GCF was collected from each subject. The GCF was transferred into vials containing $100 \mu \mathrm{l}$ phosphate buffer saline and the samples were frozen at $-70^{\circ} \mathrm{C}$ till they were assayed for ADM.

\section{Measurement of Adrenomedullin Levels}

The colorimetric assay procedure was done at the Department of Microbiology, Maratha Mandal's Nathajirao G Halgekar Dental College, Belgaum. The ADM levels were measured using a commercially available colorimetric assay kit for human ADM (ELISA kit, Bio-Medical Assay, Beijing, China).

Statistical Analyses: All data were analyzed using a software program (SPSS, version 14.0, SPSS, Chicago, IL). ANOVA was carried out to test the hypothesis of equality among the five groups for ADM, to explore which pair or pairs differed at the $5 \%$ level of significance with respect to GCF ADM levels. Multiple comparisons for ADM levels using Bonferroni test was carried out to find out which pair or pairs differ significantly. Pearson's correlation coefficient test was used to observe any correlation between the clinical parameters recorded, i.e. PPD, CAL, HbAlc and GCF ADM levels.

\section{RESULTS}

\section{ADM Concentrations}

The mean ADM levels for groups I to V were 13.64, 25.22, $33.89,36.73$ and $45.37 \mu \mathrm{mol} / 1$ (Table 1 ) respectively. To test the hypothesis of equality among the five groups for ADM, ANOVA was carried out, the results of which are tabulated in Table 2. The result of ANOVA showed that the means among various groups differed significantly. Multiple comparisons 
Table 1: Descriptive analysis showing mean values, standard deviation (SD), minimum and maximum values for ADM levels $(\mu \mathrm{mol} / \mathrm{l})$

\begin{tabular}{llllll}
\hline Groups & Mean & $\begin{array}{l}\text { Standard } \\
\text { deviation }\end{array}$ & $\begin{array}{l}\text { Standard } \\
\text { error of } \\
\text { mean }\end{array}$ & Min. & Max. \\
\hline Group I & 13.64 & 2.92 & 0.75 & 7.43 & 18.97 \\
Group II & 25.22 & 4.90 & 0.89 & 17.25 & 33.59 \\
Group III & 33.89 & 4.03 & 1.04 & 26.65 & 39.56 \\
Group IV & 36.73 & 4.37 & 1.13 & 29.51 & 43.44 \\
Group V & 45.37 & 8.32 & 2.15 & 27.79 & 60.78 \\
\hline
\end{tabular}

Table 2: Results of ANOVA test comparing the mean ADM levels ( $\mu \mathrm{mol} / \mathrm{l})$ between all the groups

\begin{tabular}{llll}
\hline Groups & $n$ & $\begin{array}{l}\text { Anova } \\
\text { F-value }\end{array}$ & $p$-value \\
\hline Group I & 15 & & \\
Group II & 30 & & \\
Group III & 15 & 85.336 & $<0.001^{*}$ \\
Group IV & 15 & & \\
Group V & 15 & & \\
\hline
\end{tabular}

$\mathrm{n}$ denotes number of samples; *Significant at p-value $<0.05$

Table 3: Group-wise comparison for ADM levels ( $\mu \mathrm{mol} / \mathrm{l})$ using Bonferroni test

\begin{tabular}{llll}
\hline Group (I) & Group (J) & Mean difference (I-J) & $p$-value \\
\hline Group I & Group II & -11.587 & $<0.001^{*}$ \\
& Group III & -20.257 & $<0.001^{*}$ \\
& Group IV & -23.096 & $<0.001^{*}$ \\
& Group V & -31.735 & $<0.001^{*}$ \\
Group II & Group III & -8.670 & $<0.001^{*}$ \\
& Group IV & -11.509 & $<0.001^{*}$ \\
& Group V & -20.148 & $<0.001^{*}$ \\
Group III & Group IV & -2.839 & 1.000 \\
& Group V & -11.478 & $<0.001^{*}$ \\
Group IV & Group V & -8.639 & $<0.001^{*}$ \\
\hline
\end{tabular}

${ }^{*}$ Significant at $p$-value $<0.05$
Table 5: Pearson correlation coefficient test comparing $\mathrm{HbA} 1 \mathrm{c}$ levels with probing pocket depth (PPD) and clinical attachment level (CAL)

\begin{tabular}{llllll}
\hline Groups & \multicolumn{2}{l}{ HbA1c and $P P D$} & & \multicolumn{2}{c}{ HbA1c and CAL } \\
\cline { 2 - 3 } \cline { 6 - 7 } & $r$ & $p$-value & & $r$ & $p$-value \\
\hline Group III & 0.037 & 0.913 & & -0.094 & 0.739 \\
Group IV & 0.347 & 0.206 & & 0.092 & 0.744 \\
Group V & 0.452 & 0.091 & & -0.074 & 0.793 \\
\hline
\end{tabular}

for ADM levels using Bonferroni test was carried out to find out which pair or pairs differ significantly. On comparing all the five groups, the difference was statistically significant between all the groups except between groups III and IV (Table 3).

\section{Correlations}

Pearson's correlation coefficient test was used to observe any correlation between the clinical parameters recorded, i.e. PPD, CAL, HbA1c and GCF ADM levels. ADM was found to be positively correlated with PPD, CAL and $\mathrm{HbA} 1 \mathrm{c}$ in all the groups except group I where a negative correlation was found between ADM and CAL. The correlations were found to be statistically significant between ADM and PPD and CAL in all the groups except in group I. The correlation was found to be statistically not significant between ADM and HbA1c in groups III, IV and V (Table 4).

Pearson's correlation coefficient test was used to observe any correlation between $\mathrm{HbA1c}$ and PPD, CAL. There was statistically no significant correlation between $\mathrm{HbA} 1 \mathrm{c}$ and PPD and CAL in groups III, IV and V. Between HbA1c and PPD, there was positive correlation in groups III, IV and $\mathrm{V}$. HbA1c and CAL in groups III and V were negatively correlated (Table 5).

\section{DISCUSSION}

Diabetes mellitus and periodontal disease share a two-way relationship. Findings from various studies indicate that diabetes mellitus leads to a hyper-inflammatory response to the periodontal microbiota and also impairs resolution

Table 4: Pearson correlation coefficient test comparing ADM levels $(\mu \mathrm{mol} / \mathrm{l})$ with probing pocket depth (PPD), clinical attachment level (CAL) and $\mathrm{HbA} 1 \mathrm{C}$

\begin{tabular}{|c|c|c|c|c|c|c|}
\hline \multirow[t]{2}{*}{ Groups } & \multicolumn{2}{|c|}{$A D M$ and $P P D$} & \multicolumn{2}{|c|}{$A D M$ and $C A L$} & \multicolumn{2}{|c|}{$A D M$ and $H b A 1 c$} \\
\hline & $r$ & $p$-value & $r$ & $p$-value & $r$ & $p$-value \\
\hline Group I & 0.051 & 0.855 & -0.083 & 0.769 & - & - \\
\hline Group II & 0.743 & $<0.001^{*}$ & 0.745 & $<0.001^{*}$ & - & - \\
\hline Group III & 0.896 & $<0.001^{*}$ & 0.824 & $<0.001^{*}$ & 0.203 & 0.468 \\
\hline Group IV & 0.701 & $0.004^{*}$ & 0.685 & $0.005^{*}$ & 0.315 & 0.252 \\
\hline Group V & 0.944 & $<0.001^{*}$ & 0.537 & $0.039^{*}$ & 0.414 & 0.125 \\
\hline
\end{tabular}

*Significant at $p$-value $<0.05$ 
of inflammation and repair, which leads to accelerated periodontal destruction. ${ }^{9,12,13}$ Periodontitis has been reported to adversely affect glycemic control in patients with diabetes mellitus and to contribute to the development of diabetic complications. The inflamed periodontium is highly vascular and the ulcerated pocket epithelium may serve as a portal to the systemic circulation for bacterial products and locally produced inflammatory mediators. ${ }^{14}$ Antimicrobial peptides including defensins, cathelicidin and ADM are important contributors for maintaining the balance between the healthy and diseased conditions of the oral cavity. ${ }^{2,3}$

Studies in the literature have evaluated levels of ADM in GCF of patients with periodontitis and serum of diabetic patients. ${ }^{15-18}$ This study was undertaken to estimate and compare the ADM levels in GCF from subjects with clinically healthy periodontium, periodontitis patients, and periodontitis patients with different glycemic controls.

Ninety patients with age ranging from 35 to 75 years were selected for the study. The prevalence of periodontal disease increases rapidly with age and also most people with type 2 diabetes belong to this age group. ${ }^{19,20}$ This age range is in accordance with other studies. ${ }^{21,22}$

Patients were divided into five groups - groups I to V. Groups III, IV, V were categorized based on their glycated hemoglobin levels. HbA1c estimates the glycemic control of patients over the preceding 3 months. ${ }^{22}$

CPI score was used for the screening of periodontal disease in this study. The CPI was developed as an initiative of World Health Organization and has been reported as the practical method for routine screening and recording of periodontal disease indicators due to its simplicity and uniformity. $^{23}$

GCF was collected from a predetermined site and colorimetric ELISA assay was performed to assess the level of ADM in all study groups. As the composition of GCF is the result of interplay between the bacterial biofilm and cells of the periodontal tissues, analysis of special constituents in the GCF provides a qualitative biochemical indicator for evaluation of local cellular metabolism that clearly reflects the current periodontal status. An added advantage in GCF collection is that, it is a noninvasive procedure as compared to gingival tissue biopsy. ${ }^{24}$ Also, the concentrations ADM are enriched about 30 -fold in gingival crevicular fluid as compared to saliva. ${ }^{25}$

In this study, GCF was collected using calibrated microcapillary pipettes. Microcapillary pipettes facilitated the collection of a standardized GCF volume of $3 \mu \mathrm{l}$ for all the subjects, as required for the biochemical analysis of GCF and also this technique is advantageous in that it allows for the analysis of an undiluted sample of GCF ADM levels. GCF was collected from the mesiobuccal region of the maxillary right first molar in healthy subjects, in the absence of which the left first molar was sampled. This site was selected as adequate fluid volume could be obtained from this site. In case of periodontitis patients, the site with deepest probing depth was selected for GCF collection.

The results of the present study reported that levels of ADM in GCF increases progressively from healthy (13.64 \pm $2.92 \mu \mathrm{mol} / \mathrm{l})$ to periodontitis subjects $(25.22 \pm 4.90 \mu \mathrm{mol} / \mathrm{l})$; and from well-controlled diabetics $(33.89 \pm 4.03 \mu \mathrm{mol} / \mathrm{l})$ to moderately-controlled $(36.73 \pm 4.37 \mu \mathrm{mol} / \mathrm{l})$ and poorly controlled type 2 diabetics with periodontitis $(45.37 \pm 8.32$ $\mu \mathrm{mol} / 1)$.

Other studies have reported increased GCF ADM level in periodontitis subjects as compared to periodontal health. ${ }^{5,15}$ The results of our study also support the trend of increasing ADM levels from periodontal health to disease.

This increase in GCF ADM level in patients with periodontitis could be because of the presence of more severe inflammation and periodontal destruction and an increase of bacteria-stimulated ADM production by epithelial cells to protect the host from bacterial invasion, all of which exhibit increased LPS-induced ADM production. ${ }^{26,27}$ Though patients with periodontitis have a significantly elevated total amount of GCF ADM, these levels were not adequate to prevent periodontal tissue destruction in the patients. This is because $P$. gingivalis, which is an important periodontopathogen for chronic periodontitis, is able to defend against the antimicrobial effect of ADM. ${ }^{28}$

The literature consists of a number of studies measuring the ADM levels in diabetic subjects using serum, plasma as samples. Turk et al examined 64 type 2 diabetic patients and 20 healthy subjects as control group and found that plasma ADM levels were significantly elevated in patients with type 2 diabetes when compared with the control group. ${ }^{18}$ Studies have shown that individuals with type 2 diabetes were at a higher risk for vascular injury due to the endothelial dysfunction and ADM which is produced by the endothelium has vasoprotective (vasodilating and anti proliferative) properties, so the rise in ADM GCF levels in type 2 diabetic patients can be due to hyperglycemia induced increase expression of ADM. ${ }^{10,16}$

In type 2 diabetes mellitus patients with periodontitis, the results of our study report a gradual increase in ADM levels progressing from well-controlled to moderately-controlled to poorly-controlled subjects. To the best of our knowledge, no other study in the literature has studied the levels of ADM in different levels of glycemic control (i.e. well-controlled, moderately- and poorly-controlled). It remains unclear whether ADM levels are influenced by diabetes severity or the circulating ADM gets influenced by the severity of periodontitis. 
The results of this study shows statistically significant relationship between ADM and PPD and CAL in all the groups except in healthy group which is in accordance with the other studies. ${ }^{15}$ The variability of ADM concentrations within the patients of each group can be attributed to the heightened protective response to increasing severity of both periodontal disease and diabetes, thus emphasizing the common multifactorial etiology and host response to both periodontal disease and diabetes mellitus. The result of this study also implies the possible role of adrenomedullin as a marker for both periodontal disease and diabetes.

The limitations of the study include the fact that it was conducted on a small sample size and that it did not eliminate the confounding influence of obesity.

\section{CONCLUSION}

Following conclusion can be drawn from the study:

1. ADM is present in GCF in periodontal health and disease.

2. There is a substantial increase in the levels of ADM in GCF in periodontitis and periodontitis with type 2 diabetes mellitus.

3. The levels of ADM increase as the glycemic control of the diabetic patient worsens.

4. Significant correlation is present between PPD and CAL and ADM levels in GCF in all the periodontitis patients with or without type 2 diabetes.

Within the limitations of the present study, it can be postulated that increased levels of ADM can be detected in GCF in periodontitis and periodontitis subjects with type 2 diabetes with worsening of glycemic control. However, further longitudinal studies with a large sample size are needed to validate $\mathrm{ADM}$ as a marker in periodontal disease and diabetes progression and to prove its protective role in periodontal disease and type 2 diabetes mellitus.

\section{REFERENCES}

1. Newman MG, Takei HH, Klokkevold PR, Caranza FA. Carranza's clinical periodontology. 9th ed. Noida. Saunders: Reed Elsevier India Private Limited 1996.

2. Altman H, Steinberg D, Porat Y, et al. In vitro assessment of antimicrobial peptides as potential agents against several oral bacteria. J Antimicrob Chemother 2006;58:198-201.

3. Bals R. Epithelial antimicrobial peptides in host defense against infection. Respir Res 2000;1:141-150.

4. Dale BA, Fredericks LP. Antimicrobial peptides in the oral environment: Expression and function in health and disease. Curr Issues Mol Biol 2005;7:119-133.

5. Lundy FT, O'Hare MM, McKibben BM, Fulton CR, Briggs JE, Linden GJ. Radioimmunoassay quantification of adrenomedullin in human gingival crevicular fluid. Arch Oral Biol 2006;51: 334-338.

6. Kapas S, Pahal K, Cruchley AT, Hagi-Pavli E, Hinson JP. Expression of adrenomedullin and its receptors in human salivary tissue. J Dent Res 2004;83:333-337.

7. Kapas S, Bansal A, Bhargava V, Maher R, Malli D, Hagi-Pavli E. Adrenomedullin expression in pathogen-challenged oral epithelial cells. Peptides 2001;22:1485-1489.
8. Elsasser TH, Kahl S. Adrenomedullin has multiple roles in disease stress: Development and remission of the inflammatory response. Microsc Res Tech 2002;57:120-129.

9. Iacopino AM. Periodontitis and diabetes interrelationships: Role of inflammation. Ann Periodontol 2001;6(1):125-137.

10. Lim SC, Morgenthaler NG, Subramaniam T, Wu YS, Goh SK, Sum CF. The relationship between adrenomedullin, metabolic factors and vascular function in individuals with type 2 diabetes. Diabetes Care 2007 Jun;30(6):1513-1519.

11. Extracts of the Fourth edition of 'Oral Health Surveys - Basic methods', Geneva 1997. Available at: http//:www.whocollab. od.mah.se/expl/orhcpitn97.html (19-07-2009).

12. Mealey BL, Ocampo GL. Diabetes mellitus and periodontal disease. Periodontology 2000. 2007;44:127-153.

13. Löe H. Periodontal disease: the sixth complication of diabetes mellitus. Diabetes Care 1993;16(1):329-334.

14. Grossi SG, Genco RJ. Periodontal disease and diabetes mellitus: a two-way relationship. Ann Periodontol 1998;3(1):51-61.

15. Turkoglu O, Emingil G, Kutukcxuler N, Atilla G. Evaluation of gingival crevicular fluid adrenomedullin and human neutrophil peptide 1-3 levels of patients with different periodontal diseases. J Periodontol 2010;81:284-291.

16. Ruzicska E, Toth M, Tulassay Z, Somogyi A. Adrenomedullin and diabetes mellitus. Diabetes Metab Res Rev 2001;17: 321-329.

17. Garcia-Unzueta MT, Montalban C, Pesquera C, Berrazueta JR, Amado JA. Plasma adrenomedullin levels in type 1 diabetes: relationship with clinical parameters. Diabetes Care 1998;21: 999-1003.

18. Turk HM, Buyukberber S, Sevinc A, et al. Relationship between plasma adrenomedullin levels and metabolic control, risk factors, and diabetic microangiopathy in patients with type 2 diabetes. Diabetes Care 2000;23:864-867.

19. Mealey B. Diabetes mellitus. In: Rose LF, Genco RJ, Mealy BL,Cohen WD, editors. Periodontal Medicine. Hamilton, Ontario: BC Decker Inc.; 2000. p. 121-151.

20. Powers AC. Diabetes mellitus. In: Braunwald E, Fauci AS, Kasper DL, Hauser SL, Longo DL, Jameson JL, editors. Harrison's Principles of Internal Medicine. 16th ed., New York: McGraw Hill Companies 2005;2:p 2137-2152.

21. Kiran M, Arpak N, Unsal E, Erdogan MF. The effect of improved periodontal health on metabolic control in type 2 diabetes mellitus. J Clin Periodontol 2005;32(3):266-272.

22. Faria-Almeida R, Navarro A, Bascones A. Clinical and metabolic changes after conventional treatment of type 2 diabetic patients with chronic periodontitis. J Periodontol 2006;77(4):591-598.

23. Nomura Y, Tamaki Y, Tanaka T, et al. Screening of periodontitis with salivary enzyme test. Journal of Oral Science 2006;48(4): 177-183.

24. Okada H, Murakami S. Cytokine expression in periodontal health and disease. Crit Rev Oral Biol Med 1998;9(3):248-266.

25. Sven-Ulrik Gorr. Antimicrobial peptides of the oral cavity. Periodontology 2000. 2009;51:152-180.

26. Hosokawa I, Hosokawa Y, Ozaki K, Nakae H, Matsuo T. Adrenomedullin suppresses tumour necrosis factor alphainduced CXC chemokine ligand 10 production by human gingival fibroblasts. Clin Exp Immunol 2008;152:568-575.

27. Isumi $Y$, Minamino $N$, Katafuchi $T$. Adrenomedullin production in fibroblasts: Its possible function as a growth regulator of Swiss 3T3 cells. Endocrinology. 1998;139:2552-2563.

28. Allaker RP, Sheehan BE, McAnerney DC, McKay IJ. Interaction of adrenomedullin and calcitonin gene-related peptide with the periodontal pathogen porphyromonas gingivalis. FEMS Immunol Med Microbiol 2007;49:91-97. 